\title{
Cancer Dormancy: A New Hope
}

\author{
Surajit Sinha* \\ Center for Cancer Research, National Cancer Institute, USA
}

\begin{abstract}
Metastasis is the major cause of cancer-related deaths. Many patients develop metastatic relapse in secondary organs years after resection of the primary tumor, and usually succumb to the disease. The most likely explanation for this clinical outcome is that the early dissemination of tumor cells in the systemic circulation and seeding of secondary organs had occurred prior to the primary tumor resection and that disseminated tumor cells (DTCs) remained growth arrested in target organs for extended periods before the relapse. This asymptomatic phase of growth arrest before reactivation and manifestation of metastatic disease is called 'tumor dormancy'.
\end{abstract}

Although most driver genetic alterations, like the RAS mutation (in pancreatic cancer), ERBB2 amplification (in breast cancer) and androgen receptor amplification (in prostate cancer), can initiate primary tumor growth and dissemination, they are insufficient to drive metastatic outgrowth by themselves. Thus, during the dormancy phase, DTCs evolve, undergo additional molecular changes at both the genetic and epigenetic levels, engage or hijack growth-permissive signaling mechanisms of the host organ, gain competence and become metastasis-initiating cells (MICs), therebypropelling metastatic outgrowth. ${ }^{1-3}$ Since metastatic tumors are highly recalcitrant to chemotherapeutic drugs and are usually untreatable (eventually compromising the function of the organ), adjuvant treatments targeting DTCs or MICs during the dormancy phase can provide better therapeutic response and patient survival.

The DTC- or MIC-targeted approach provides the best window of opportunity for elimination of residual disease. However, understanding the biology of residual disease has been a challenge, mainly because of the lack of mouse models that can recapitulate the complex metastatic cascade of the human disease process. Previous studies have shown that targeted therapies or adjuvant treatments are largely ineffective in eliminating residual DTCs from the system because the biology and molecular signatures of disseminated disease is different from that of primary tumor, hinting at an indispensable need for development of mouse models of dormancy and metastasis.

A recent flurry of investigations using genetic screening in mice and dormant cell lines isolated from patients have provided some insights into the signaling mechanisms that govern metastatic dormancy and reactivation. ${ }^{4,5}$ This has raised and fostered hope that dissecting the biology and mechanisms that regulate dormancy and reactivation of tumor cells will lead to development of biomarkers

\footnotetext{
Abbreviations: DTC, disseminated tumor cells; MIC, metastasis initiating cells; GAS, growth arrest specific; BMP, bone morphogenetic protein; TGF, transforming growth factor.

Received: May 02, 2017; Revised: September 08, 2017; Accepted: September 22, 2017

*Correspondence to: Surajit Sinha, Center for Cancer Research, National Cancer Institute, USA. Tel: +1-917-5366679, E-mail: surajit.sinha@nih.gov

How to cite this article: Sinha S. Cancer Dormancy: A New Hope. Exploratory Research and Hypothesis in Medicine 2018;3(1):1-3. doi: 10.14218/ERHM.2017.00011.
}

that can aid in determining the extent of metastatic spread and in rationalizing patient treatment; in addition, it may also ultimately lead to improvement of existing treatments and to design of novel therapeutic modalities for eradication and complete remission of the cancer. ${ }^{6}$

MICs arise from DTCs that detach from the primary tumor and enter the systemic circulation. Upon extravasation into a target organ, such as liver or lung, the majority of DTCs undergo rapid apoptosis and clearance because of mechanical/metabolic stresses and immune surveillance by the host. Few DTCs survive this attrition process and undergo extended growth arrest, and even fewer cells survive, gain competence and initiate metastatic outgrowth. As such, the entire metastatic cascade is a Darwinian selection process, wherein the majority of DTCs in the circulation and during extravasation are eliminated as they are not deemed fit. Only a very few survive the entire attrition process to develop into lifethreatening lesions.

Emerging studies show that these few life-threatening lesions develop from cancer cells that have undergone reprogramming to become stem-like and exhibit high self-renewal properties that are just like normal somatic stem cells. ${ }^{7-9}$ Why DTCs become dormant and how they gain metastatic competence to re-initiate outgrowth at a distant organ is not known. The question arises then, can effective therapies be designed to eliminate disseminated residual cells? Such questions are only just beginning to be understood at the molecular level.

One hypothesis is that the absence of or reduced mitogenic signaling in the secondary organ can trigger tumor cells to undergo a G0/G1 arrest. In such scenarios, tumor cells are unable to engage growth factor signaling through integrin receptors and enter the state of dormancy or quiescence. Most organs in the human body have a growth-restrictive or rather dormancy-permissive microenvironment to any foreign tumor cell that has infiltrated the tissue. For example, in the bone marrow, growth arrest-specific protein 6 (GAS6) and specific bone morphogenetic protein (BMP7) can growth-arrest tumor cells and induce dormancy in leukemia and prostate cancers. ${ }^{10-12}$ In the lungs, the abundance of BMP4, and similarly the anti-proliferative effect of transforming growth factor-beta 2 (TGF- $\beta 2$ ), in the bone marrow can restrict the growth of DTCs and induce quiescence..$^{13,14}$

The second hypothesis involves the inability of tumor cells to mount an angiogenic switch. Without a constant supply of nutrients and in the absence of newly formed blood vessels, a balance is maintained between proliferation and apoptosis, leading to a state of dormancy. ${ }^{15}$ It has recently been theorized that dormancy is an active molecular program adopted by DTCs, just like quiescence of normal stem cells. This theory was supported by a study that demonstrated a significant overlap in the molecular programs that induce quiescence in hair and muscle stem cells with those of dormant head and neck carcinoma cells. ${ }^{16}$

Recent investigations into the mechanisms of dormancy and 


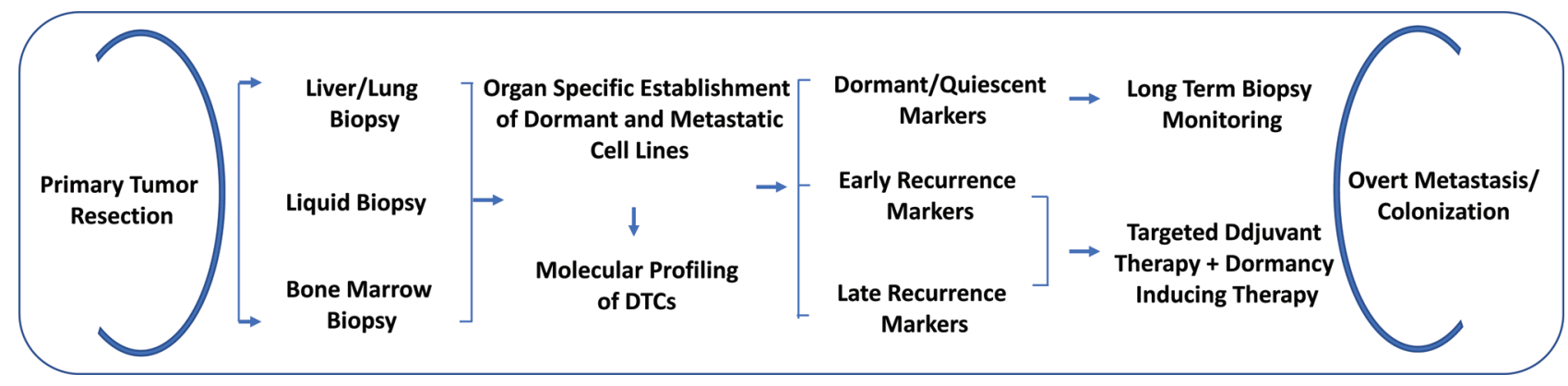

Fig. 1. Schematic showing how understanding the molecular features of disseminated tumor cells from various biopsies can aid in future control and treatment of disseminated residual disease before their development into overt metastasis. Isolation and profiling of disseminated and dormant tumor cells from adjacent normal tissue of patients undergoing liver/lung tumor resection (the adjacent normal tissue near the tumorsite harbor disseminated tumor cells which have homed the organ but did not colonize) or liquid biopsies \{circulating tumor cells (CTCs) and circulating tumor DNAs (ctDNA) or disseminated tumor cells(DTCs) from bone marrow of patients undergoing primary tumor resection will help in generating dormancy and recurrence markers. These markers can then be used to monitor metastatic progression and appropriate adjuvant cocktails can be designed to block or reduce recurrence markers and induce perpetual dormancy signature.

metastatic reactivation have demonstrated that dormant tumor cells activate intrinsic molecular programs that induce stem cell traits or self-renewal capacity to colonize a target organ. Moreover, genetic screening in mouse models and mechanistic work have identified tumor intrinsic factors and receptors that can cross-talk with the tumor microenvironment to initiate metastatic outgrowth. $4,17,18$

A main question that persists is how understanding dormancy will improve patient treatment. First, the dissection of molecular mechanisms that dictate dormancy will greatly accelerate development of novel biomarkers that can distinguish early and late DTCs and generate dormancy gene signatures that can predict disease recurrence. Such markers can be used for monitoring metastatic progression. Adjuvant treatment of patients can then be stratified based on the risk of disease recurrence. Second, most conventional chemotherapeutic drugs are administered to induce cancer cell death but they also generate drug-resistant cells, which become untreatable. In contrast, however, adjuvant treatments of patients with a cocktail of mitogenic signaling inhibitors can reprogram a metastatic initiating cell to enter perpetual dormancy. Third, dormant DTCs, which are supposedly cancer stem cells, although growth arrested in nature, have functional metabolically active survival pathways, such as autophagy, which can be targeted.

Understanding the metabolic and survival pathways at singlecell level will allow rational intervention to induce metabolic dormancy. It is, therefore,imperative to establish the genomic and proteomic profiles of dormant versus MICs to strategically intervene these processes. Thus, characterization of dormant and DTCs may not only be the best and quickest method of determining the timing of disease recurrence or extent of dormancy but may also help in rationalizing adjuvant treatment to delay the onset of recurrence or to drive DTCs into perpetual dormancy (Fig. 1).

Our knowledge on cancer dormancy is expanding fast, and application of this knowledge in the clinic is slowly budding. RNA sequencing technology at single-cell level has given a boom to this field and has helped in characterization of DTCs from bone marrow and circulating tumor cells from liquid biopsies. As most solid cancers are organotropic, it is the task of future scientists to develop organ-specific biomarkers for DTCs. It is also imperative to develop proteomic profiles of DTCs as proteins are the drivers of phenotype. Together, this knowledge will help in proper staging and stratification of cancer patients for adjuvant therapy. Although the field of cancer dormancy is currently in its infancy and many technological challenges remain, it holds great potential and promise for future cancer treatments and patient management, and if successful can establish a paradigm for next-generation treatment modalities.

\section{References}

[1] Sosa MS, Bragado P, Aguirre-Ghiso JA. Mechanisms of disseminated cancer cell dormancy: an awakening field. Nat RevCancer 2014;14(9):611-622. doi:10.1038/nrc3793.

[2] Hosseini H, Obradović MM, Hoffmann M, Harper KL, Sosa MS, Werner-Klein $\mathrm{M}$, et al. Early dissemination seeds metastasis in breast cancer. Nature 2016;540:552-558. doi:10.1038/nature 20785.

[3] Massagué J, Obenauf AC. Metastatic colonization by circulating tumour cells. Nature 2016;529(7586):298-306. doi:10.1038/nature 17038.

[4] Gao H, Chakraborty G, Lee-Lim AP, Mavrakis KJ, Wendel HG, Giancotti FG. Forward genetic screens in mice uncover mediators and suppressors of metastatic reactivation. Proc Natl AcadSci U S A 2014;111(46):16532-16537. doi:10.1073/pnas.1403234111.

[5] Giancotti FG. Mechanisms governing metastatic dormancy and reactivation. Cell 2013;155(4):750-764. doi:10.1016/j.cell.2013.10.029.

[6] Aguirre-Ghiso JA, Bragado P, Sosa MS. Metastasis awakening: targeting dormant cancer. Nat Med 2013;19(3):276-277. doi:10.1038/ nm.3120.

[7] Linde N, Fluegen G, Aguirre-Ghiso JA. The relationship between dormant cancer cells and their microenvironment. Adv Cancer Res 2016;132:45-71. doi:10.1016/bs.acr.2016.07.002.

[8] Fluegen G, Avivar-Valderas A, Wang Y, Padgen MR, Williams JK, Nobre $A R$, et al. Phenotypic heterogeneity of disseminated tumour cells is preset by primary tumour hypoxic microenvironments. Nat Cell Biol 2017;19(2):120-132. doi:10.1038/ncb3465.

[9] Nguyen DX, Bos PD, Massagué J. Metastasis: from dissemination to organ-specific colonization. Nat Rev Cancer 2009;9(4):274-284. doi:10.1038/nrc2622.

[10] Dormady SP, Zhang XM, Basch RS. Hematopoietic progenitor cells grow on $3 \mathrm{~T} 3$ fibroblast monolayers that overexpress growth arrestspecific gene-6 (GAS6). Proc Natl Acad Sci U S A 2000;97(22):1226012265. doi:10.1073/pnas.97.22.12260.

[11] Taichman RS, Patel LR, Bedenis R, Wang J, Weidner S, Schumann T, et al. GAS6 receptor status is associated with dormancy and bone metastatic tumor formation. PLoS One 2013;8(4):e61873. doi:10.1371/ journal.pone.0061873.

[12] Kobayashi A, Okuda H, Xing F, Pandey PR, Watabe M, Hirota S, et al. Bone morphogenetic protein 7 in dormancy and metastasis of pros- 
tate cancer stem-like cells in bone. J Exp Med 2011;208(13):26412655. doi:10.1084/jem.20110840.

[13] Gao H, Chakraborty G, Lee-Lim AP, Mo Q, Decker M, Vonica A, et al. The BMP inhibitor Coco reactivates breast cancer cells at lung metastatic sites. Cell 2012;150(4):764-779. doi:10.1016/j. cell.2012.06.035.

[14] Bragado P, Estrada Y, Parikh F, Krause S, Capobianco C, Farina $\mathrm{HG}$, et al. TGF- $\beta 2$ dictates disseminated tumour cell fate in target organs through TGF- $\beta$-RIII and $p 38 \alpha / \beta$ signaling. Nat Cell Biol 2013;15(11):1351-1361. doi:10.1038/ncb2861.

[15] Ghajar CM, Peinado H, Mori H, Matei IR, Evason KJ, Brazier H, et al.
The perivascular niche regulates breast tumour dormancy. Nat Cell Biol 2013;15(7):807-817. doi:10.1038/ncb2767.

[16] Cheung TH, Rando TA. Molecular regulation of stem cell quiescence. Nat Rev Mol Cell Biol 2013;14(6):329-340. doi:10.1038/nrm3591.

[17] Gao H, Chakraborty G, Zhang Z, Akalay I, Gadiya M, Gao Y, et al Multi-organ site metastatic reactivation mediated by non-canonical discoidin domain receptor 1 signaling. Cell 2016;166(1):47-62. doi:10.1016/j.cell.2016.06.009.

[18] Bragado P, Sosa MS, Keely P, Condeelis J, Aguirre-Ghiso JA. Microenvironments dictating tumor cell dormancy. Recent Results Cancer Res 2012;195:25-39. doi:10.1007/978-3-642-28160-0_3. 